\title{
Adiciones y correcciones a la flora bentónica marina del Atlántico ibérico norte
}

Ignacio Bárbara, Verónica García-Redondo, Pilar Díaz Tapia, Alicia García-Fernández, Cristina Piñeiro-Corbeira, Viviana Peña, Juan Lugilde \& Javier Cremades

BioCost, Facultad de Ciencias \& CICA, Universidade da Coruña, Campus de la Zapateira, 15071. A Coruña

\section{Correspondencia}

I. Bárbara

e-mail: barbara@udc.es

Recibido: 3 marzo 2019

Aceptado: 22 mayo 2019

Publicado on-line: 18 septiembre 2019

Editado por: E. Bañares

\section{New records for the benthic marine flora of Atlantic Iberia}

Palabras clave: Algas marinas, distribución, nuevas citas, Península lbérica

Key words: Distribution, Iberian Peninsula, new record, seaweeds
Continuando con los estudios para la actualización del inventariado de la flora bentónica marina del Atlántico Ibérico (Bárbara et al. 2005a-b, 2006, 2008, 2012, 2014, 2015, 2016), se presentan nuevas citas y correcciones florísticas que completan el actual inventario de la biodiversidad marina de Galicia (Bañón 2017). La muestras se conservaron en formalina al $4 \%$ y la conservación definitiva se realizó en pliegos de herbario, depositados en el herbario de la Universidad de Santiago de Compostela (SANT), así como en preparaciones semipermanentes en Karo®. También se estudiaron pliegos de herbario del Nationaal Herbarium Nederland, para la verificación o corrección de citas antiguas. Paralelamente, se conservaron fragmentos en fresco de algunas especies en gel de sílice para estudios moleculares.

En este trabajo se aporta información novedosa sobre la distribución de 19 especies (3 Cyanobacteria, 9 Rhodophyta, 5 Ochrophyta, 2 Chlorophyta) de algas bentónicas marinas, recolectadas en el intermareal y submareal en 51 localidades del atlántico ibérico norte. Atendiendo a la distribución de las especies recolectadas, cabe destacar una nueva cita para Europa (Plocamium cf. ovicorne), 3 nuevas citas para Galicia (Calothrix consociata, Lyngbya martensiana y Centroceras gasparrini), 14 nuevas citas provinciales y 11 segundas citas provinciales. Además dos especies (Spermothamnion strictumy Punctaria plantaginea) son excluidas de la flora de Galicia después de estudiar el único material de herbario testigo de las citas.
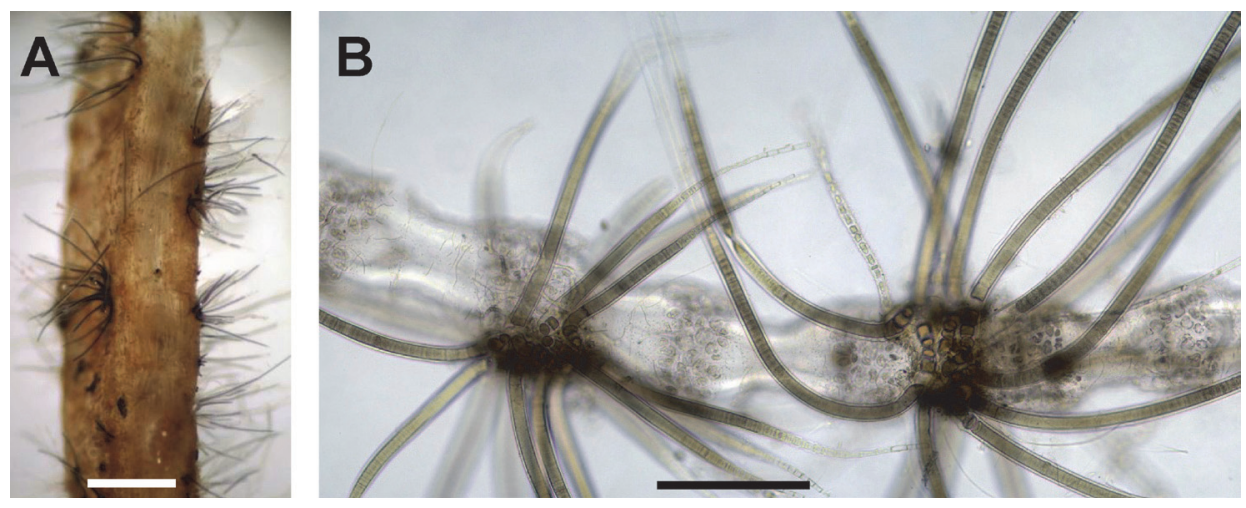

Figura 1. A-B. Calothrix consociata (Ría de Ares y Betanzos), A: sobre Cutleria multifida, B: Filamentos gregarios curvados en la base. Escalas: $\mathrm{A}=500 \mu \mathrm{m}, \mathrm{B}=200 \mu \mathrm{m}$.

Figure 1. A-B. Calothrix consociata (Ría de Ares y Betanzos), A: Close-up of colonies on Cutleria multifida, B: Gregarious filaments curved at the base. Scale bars: $A=500 \mu \mathrm{m}, \mathrm{B}=200 \mu \mathrm{m}$. 


\section{NUEVAS CITAS}

\section{CYANOBACTERIA}

\section{Calothrix consociata Bornet \& Flahault (Fig. 1)}

A CORUÑA: Ría de Ares y Betanzos, Playa de Ares, frente al río, 29TNJ616086, 22-VII-2014, 13IX-2014, submareal (1 m) sobre Cutleria multifida, Ceramium secundatum y Zostera marina, V. García-Redondo \& I. Bárbara. SANT-Algae 29097, 29274, 31778. Playa de Ares, 29TNJ613082, 17VI-2014, submareal (1 m) sobre Chondria capillaris en pradera de Z. marina, V. García-Redondo \& I. Bárbara, SANT-Algae 29289. Primera cita para Galicia.

\section{Lyngbya martensiana Meneghini ex Gomont}

A CORUÑA: Ría de Muros, ensenada de San Francisco, 29TMH949339, 8-III-2016, submareal (2 m) sobre esponja en comunidad de Cystoseira baccata, A. García-Fernández, I. Bárbara \& V. García-Redondo, SANT-Algae 31368. Primera cita para Galicia, ya que los registros anteriores (LópezRodríguez \& Pérez-Cirera 1996, Calvo et al. 1999) corresponden con Lyngbya aestuarii (Bárbara et al. 2005a).

\section{Pseudanabaena persicina (Reinke ex Gomont) Anagnostidis \\ A CORUÑA: Al sur de Illa do Valdabal, Islas de} San Pedro, A Coruña, 29TNJ444027, 23-V-2018, submareal $(3 \mathrm{~m})$ sobre Asparagopsis armata, en comunidad degradada de Cystoseira baccata y Laminaria ochroleuca, I. Bárbara \& A. GarcíaFernández, SANT-Algae 32472, 32596. Primera cita provincial y segunda para Galicia después de Bárbara et al. (2016).

\section{RHODOPHYTA}

\section{Aphanocladia stichidiosa (Funk) Ardré}

PONTEVEDRA: Ría de Arousa, Isla de A Toxa, Estruxeira, 29TNH133044, 3-VII-2015, submareal (1 m) sobre Ulva australis en pradera de Zostera marina, V. García-Redondo \& I. Bárbara, SANTAlgae 31853. Ría de Arousa, Isla de A Toxa, Os Baños, 29TNH128033, 3-VII-2015, submareal (1 m) en pradera de $Z$. marina, V. García-Redondo \& I. Bárbara, SANT-Algae 31866. Primera cita provincial.

\section{Callithamnion corymbosum (Smith) Lyngbye}

LUGO: Ría do Barqueiro, Playa de Caolín, 29TPJ067441, 8-V-2015 submareal $(3 \mathrm{~m})$ en pradera de Zostera marina, V. García-Redondo \& I. Bárbara, con espermacios, SANT-Algae 31938.
Ría do Barqueiro, Punta da Cova, 29TPJ679445, 30-V-2017, submareal (4 m) sobre Cystoseira baccata, A. García-Fernández \& I. Bárbara, SANT-Algae 32385. Ría de Viveiro, O Cargadeiro, 29TPJ122387, 19-VII-2018, submareal (4 m), sobre roca, en comunidad de $C$. baccata, A. García-Fernández \& I. Bárbara, SANT-Algae 33035. Primera cita provincial.

\section{Caulacanthus okamurae Yamada (Fig. 2)}

GUIPÚZCOA: Cabo Higuer, 30TWP978052, 5-VIII-2014, intermareal superior, sobre roca y en la base de Fucus spiralis var. limitaneus, junto a Gelidium pusillum var. pulvinatum, I. Bárbara, SANT-Algae 29054, 29055. Primera cita provincial. ASTURIAS: Puerto de Vega, 29TPJ901262, 14VIII-2014, intermareal inferior, sobre escollera del puerto, I. Bárbara, SANT-Algae 29057. Playa de Arnao, 29TPJ597240, 29-VI-2013, desde el intermareal superior al inferior, junto a Lichina pygmaea, sobre $F$. vesiculosus var. compressus y sobre roca con arena y junto a Gymnogongrus griffithsiae, SANT-Algae 29024-29026. Ría de Ribadeo, Playa de Figueras, 29TPJ596221, 22IV-2016, pantalán del puerto, junto a Polysiphonia atlantica, I. Bárbara \& V. García-Redondo, SANTAlgae 30915, 30916. Primera cita provincial. LUGO: Peinzás, Fazouro, 29TPJ396277, 22-V2016, intermareal medio, sobre F. vesiculosus var. compressus, I. Bárbara, V. Peña, J. Lugilde \& H. Kawai, SANT-Algae 30567. Ensenada de Llas, Foz, 29TPJ411264, 8-II-2016, intermareal superior, sobre roca junto a Chthamalus y L. pygmaea, I. Bárbara, SANT-Algae 30310. Ría de Viveiro, canal de entrada al puerto, 29TPJ129367, 22-V2015, intermareal inferior, sobre Mytilus, SANTAlgae 29565. Covas, Ría de Vivero, 29TPJ119367, 8-II-2016, intermareal superior, sobre F. guiryi en ambiente de roca con arena, I. Bárbara, SANTAlgae 30313. Primera cita provincial. A CORUÑA: Castillo de San Felipe, Ría de Ferrol, 29TNJ583127, 9-V-2012, intermareal medio, sobre F. vesiculosus, I. Bárbara, SANT-Algae 27405. Playa de San Pedro de Veigue, Ría de Ares y Betanzos, 29TNJ577039, 14-III-2013, intermareal medio y superior, sobre roca, Mytilus y Chthamalus, junto a Caulacanthus ustulatus y Osmundea pinnatifida, I. Bárbara, SANT-Algae 27936-27939. Al norte de Isla Castelo, Ría de A Coruña, 29TNJ530017, 11-III-2012, intermareal superior y medio, sobre Mytilus, Chthamalus, F. serratus y F. vesiculosus, junto a C. ustulatus y L. pygmaea, I. Bárbara, SANT-Algae 27378-27381. Fonteculler, Ría de A Coruña, 29TNH507975, 22-I-2012, intermareal medio, sobre Mytilus, I. Bárbara, SANT-Algae 27298. Puerto de Camelle, 29TMH927817, 13- 

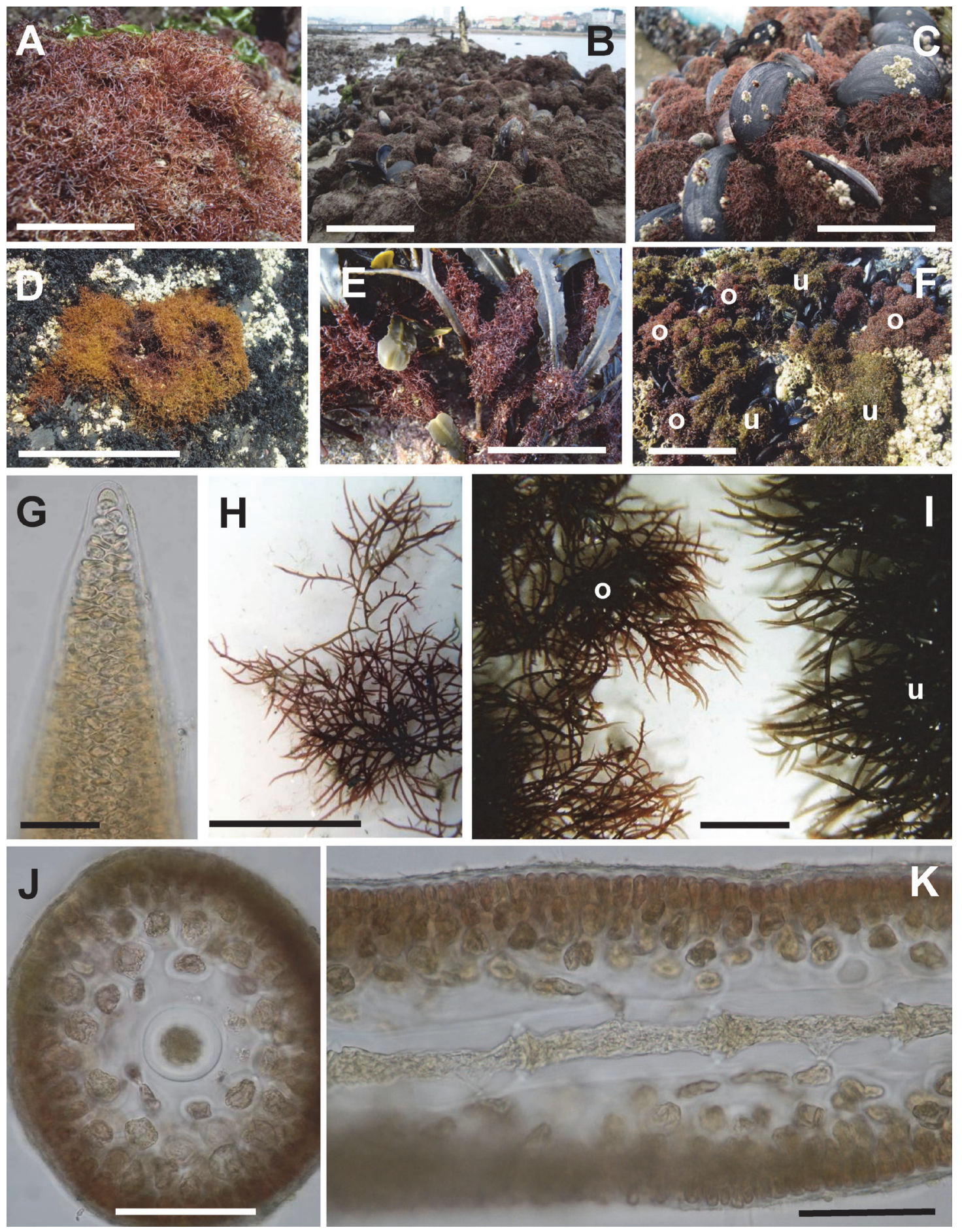

Figura 2. A-K. Caulacanthus okamurae, A: hábito sobre roca con arena (puerto de Viveiro), B-C: sobre Mytilus (Ría de A Coruña), D: sobre Chthamauls, junto a Lichina pygmaea (Ría de A Coruña), E: epifito de la base de Fucus serratus (puerto de Camelle), F: sobre Chthamalus y Mytilus, junto a $C$. ustulatus (Ría de A Coruña), G: ápice, H-I: hábito de $C$. okamurae (o) y C. ustulatus (u), J: sección transversal, K: sección longitudinal. Escalas: A-F $=10 \mathrm{~cm}, \mathrm{G}=50 \mu \mathrm{m}, \mathrm{H}, \mathrm{I}=$ $5 \mathrm{~mm}, \mathrm{~J}, \mathrm{~K}=100 \mu \mathrm{m}$.

Figure 2. A-K. Caulacanthus okamurae, A: habit overgrowing rocks with sand (harbour of Viveiro), B-C: on Mytilus (Ría de A Coruña), D: on Chthamalus, together with Lichina pygmaea (Ría de A Coruña), E: on the base of Fucus serratus (harbour of Camelle), F: on Chthamalus and Mytilus, together with $C$. ustulatus (Ría de A Coruña), G: tip, H-I: habit of $C$. okamurae (o) and C. ustulatus (u), J: cross section, K: longitudinal section. Scale bars: $A-F=10 \mathrm{~cm}, \mathrm{G}=50 \mu \mathrm{m}, \mathrm{H}, \mathrm{I}=5$ $\mathrm{mm}, \mathrm{J}, \mathrm{K}=100 \mu \mathrm{m}$. 
I-2012, intermareal medio, en la base de $F$. vesiculosus, I. Bárbara, SANT-Algae 27356. Puerto de Corrubedo, 29TMH941133, 3-III-2013, intermareal superior, sobre Chthamalus, junto a $L$. pygmaea, I. Bárbara, SANT-Algae 27868. Esteiro, Ría de Muros e Noia, 29TNH027371, 3-XII-2013, intermareal medio, junto a Mastocarpus stellatus, F. vesiculosus y Gelidium pulchellum, I. Bárbara \& P. Díaz-Tapia, SANT-Algae 28676-28678. Playa de Mañons, Ría de Arousa, 29TNH126195, 27-VIII2013, intermareal medio, sobre Mytilus, I. Bárbara,
SANT-Algae 28695. Segunda cita provincial después de Gallardo et al. (2016). PONTEVEDRA: Tragove, Ría de Arousa, 29TNH143082, 8-III2012, intermareal medio, sobre Mytilus, junto a C. ustulatus, J. Cremades, SANT-Algae 27375. Marisma entre Cambados y Tragove, Ría de Arousa, 29TNH145077, 18-V-2012, intermareal medio, sobre F. vesiculosus y Mytilus, I. Bárbara, SANT-Algae 27389, 27391. Isla de A Toxa, Ría de Arousa, 29TNH121048, 24-IV-2013, intermareal medio, sobre F. spiralis, P. Díaz-Tapia, V. García-
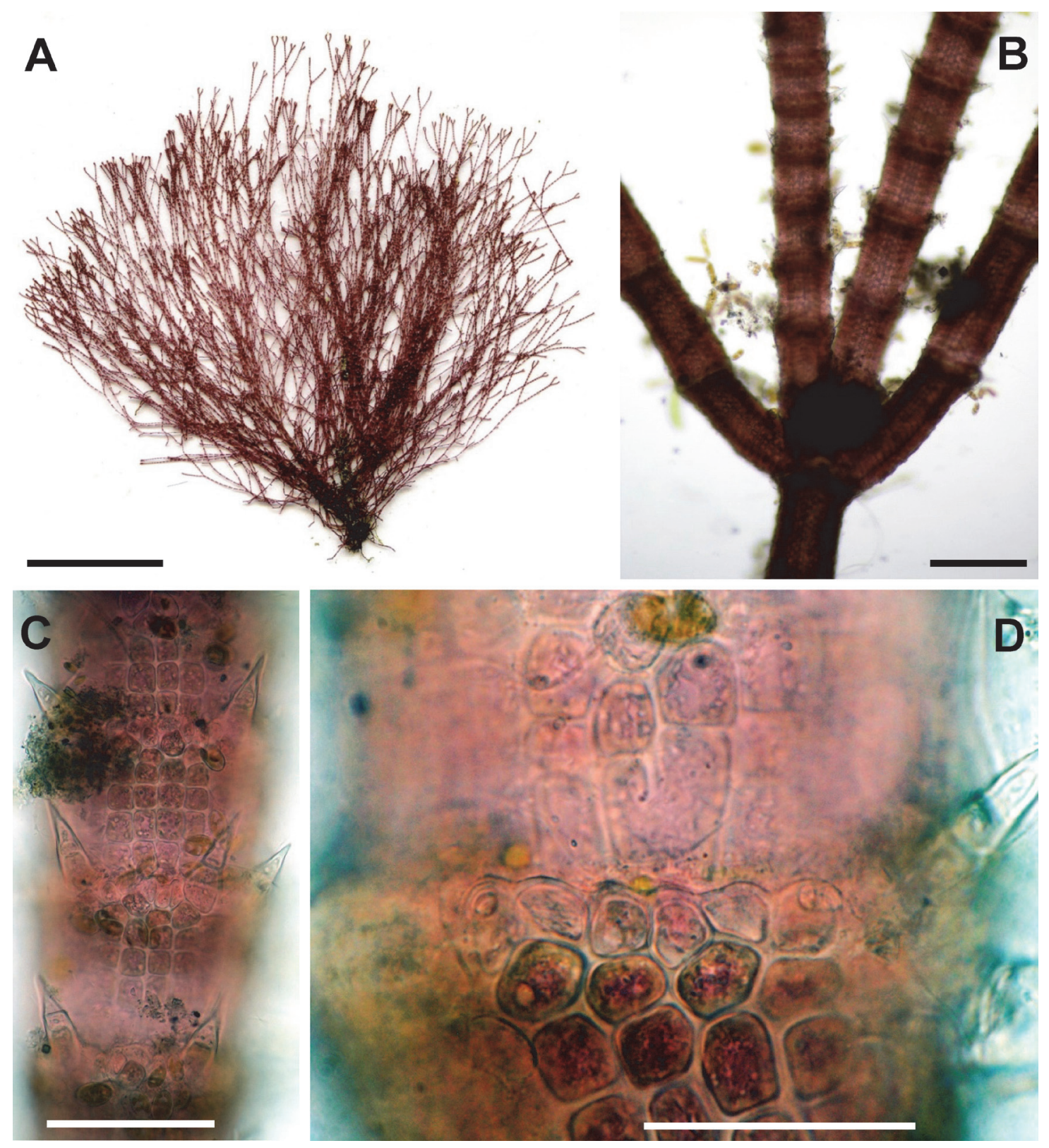

Figura 3. A-D. Centroceras gasparrinii, A: hábito, B: ramificación, C, D: corticación y verticilos de espinas. Escalas: A $=1 \mathrm{~cm}, \mathrm{~B}=200 \mu \mathrm{m}, \mathrm{C}=100 \mu, \mathrm{D}=50 \mu \mathrm{m}$.

Figure 3. A-D. Centroceras gasparrinii, A: habit, B: branching, C, D: cortication and spines disposed in whorls. Scale bars: $\mathrm{A}=1 \mathrm{~cm}, \mathrm{~B}=200 \mu \mathrm{m}, \mathrm{C}=100 \mu, \mathrm{D}=50 \mu \mathrm{m}$. 
Redondo \& C. Piñeiro, SANT-Algae 28099. Puerto de Meloxo, ría de Arousa, 29TNH089044, 24IV-2013, intermareal medio, junto a Catenella caespitosa, P. Díaz-Tapia, V. García-Redondo, C. Piñeiro \& F. Cannatà, SANT-Algae 28104. Illa de Arousa, Ría de Arousa, 29TNH116117, 23VIII-2013, intermareal medio, sobre F. serratus, I. Bárbara, SANT-Algae 28733. Playa Portomayor, Ría de Pontevedra, 29TNG194872, 9-VIII2012, intermareal medio, sobre Mytilus, junto a C. ustullatus, I. Bárbara, SANT-Algae 27554. Puente de Rande, Ría de Vigo, 29TNG283824, 1-IV-2017, intermareal medio, sobre M. stellatus y Ahnfeltiopsis devoniensis, I. Bárbara, SANTAlgae 31359. Entre Monte Ferro y Playa de Patos, 29TNG136670, 16-V-2016, sobre roca en el intermareal medio y superior, junto a $L$. pygmaea, I. Bárbara, SANT-Algae 30590-30591. Playa dos Frades, Ría de Bayona, 29TNG122637, 15-V2016, intermareal superior, junto a L. pygmaea, I. Bárbara, SANT-Algae 30911. Primera cita provincial. MINHO: Moledo, 29TNG103321, 21VIII-2013, intermareal medio, sobre Mytilus, junto a Ulva sp., I. Bárbara, SANT-Algae 28697. Forte do Cao, 29TNG104273, 22-VIII-2013, intermareal medio-superior, sobre Mytilus y L. pygmaea, I. Bárbara, SANT-Algae 28711. Primera cita provincial. BEIRA LITORAL: Buarcos, Cementera, 29TNE079475, 22-VIII-2013, intermareal medio, sobre Mytilus, I. Bárbara, SANT-Algae 28642. Primera cita provincial. En Díaz-Tapia (2014) se confirmó molecularmente la diferenciación entre C. okamurae (especies alóctona) y C. ustulatus (especie nativa), empleando secuencias del gen $r b c \mathrm{~L}$ ( $8,7 \%$ de divergencia).

\section{Centroceras gasparrinii (Meneghini) Kützing (Fig. 3)}

PONTEVEDRA: Ría de Arousa, Punta de Tragove, 29TNH142077, 26-X-2011, 5-VII-2012, intermareal medio e inferior, enredado sobre Lomentaria orcadensis, J. Cremades \& I. Bárbara, SANT-Algae 27294, 28138. Ría de Arousa, Isla de A Toxa, Estruxeira, 29TNH133044, 3-VI-2015, submareal $(1 \mathrm{~m})$, en pradera de Zostera marina, junto a Dasysiphonia japonica, V. García-Redondo \& I. Bárbara, SANT-Algae 31854. Isla de A Toxa, Os Baños, 29TNH128033, 3-VII-2015, Submareal $(1 \mathrm{~m})$ en pradera de Z. marina, junto a Cladophora lehmanniana, V. García-Redondo \& I. Bárbara, SANT-Algae 31873. Primera cita para Galicia. Coincide con la descripción de Won et al. (2009) $\mathrm{y}$ ha sido confirmado con secuencias del gen $r b c \mathrm{~L}$, presentando un $100 \%$ de similitud respecto a especímenes de la costa del Pacífico (Díaz-Tapia 2014).
Grateloupia subpectinata Holmes

A CORUÑA: Ría de Betanzos, El Pedrido, 29TNH640977, 20-VI-2009, sobre conchas y cascajo en el fango, I. Bárbara, SANT-Algae 24487, 24488. Primera cita provincial.

Nothokallymenia crouaniorum (Vergés \& Le Gall) Vergés \& Le Gall

LUGO: Ría do Barqueiro, Punta da Cova, 29TPJ679445, 30-V-2017, submareal (4 m), sobre roca junto a Kallymenia reniformis y bajo Cystoseira baccata, A. García-Fernández \& I. Bárbara. SANTAlgae 32467. Ría de Viveiro, O Cargadeiro, 29TPJ122387, 19-VII-2018, submareal (4 m), sobre roca, en comunidad de $C$. baccata, A. García Fernández \& I. Bárbara, SANT-Algae 33137. Primera cita provincial.

\section{Mesophyllum expansum (Philippi) Cabioch \& Mendoza}

LUGO: Ría de Ribadeo, Isla Pancha, 29TPJ581240, 12-VII-2018, submareal (2 m), sobre roca bajo $C$. baccata, A. García-Fernández \& I. Bárbara, SANT-Algae 33126. Pena Orxal, Cangas de Foz, 29TPJ356316, 9-VI-2017, submareal (2 m), sobre roca, bajo C. baccata, A. GarcíaFernández \& I. Bárbara, SANT-Algae 33123. Ría de Viveiro, O Cargadeiro, 29TPJ122387, 19-VII-2018, submareal (3 m), sobre roca, bajo C. baccata, A. García-Fernández \& I. Bárbara, SANT-Algae 33125. Ría do Barqueiro, Punta da Cova, 29TPJ679445, 30-V-2017, submareal (4 m) sobre roca, bajo C. baccata, A. García-Fernández \& I. Bárbara, SANT-Algae 32978. Segunda cita provincial después de Bárbara et al. (2016).

\section{Myriogramme minuta Kylin}

LUGO: Ría de Viveiro, O Cargadeiro, 29TPJ122387, 19-VII-2018, submareal (4 m), sobre roca, en comunidad de Cystoseira baccata, A. García Fernández \& I. Bárbara, SANT-Algae 33139. Segunda cita provincial después de Cremades et al. (1997).

\section{Plocamium cf. ovicorne Okamura (Fig. 4)}

A CORUÑA: Al Este del Segaño, Ría de Ferrol, 29TNJ565115, 13-Vl-2014, submareal (1 m) enredado en la base de Zostera marina, I. Bárbara \& V. García-Redondo, SANT-Algae 29111. Piedra de la Reina, Ría de Ferrol, 29TNJ569116, 27-I-2015, 20-II-2015, 19-III-2015, 16-IV-2015, 15-V-2015, 15-VI-2015, 14-VIII-2015, 13-VIII-2015, 14-X-2015, 11-XI-2015, 11-XII-2015, 15-I-2016, submareal (1 $\mathrm{m})$, enredado en la base de $Z$. marina, junto con Bonnemaisonia hamifera, Dasysihonia japonica y Plocamium cartilagineum, V. García-Redondo 

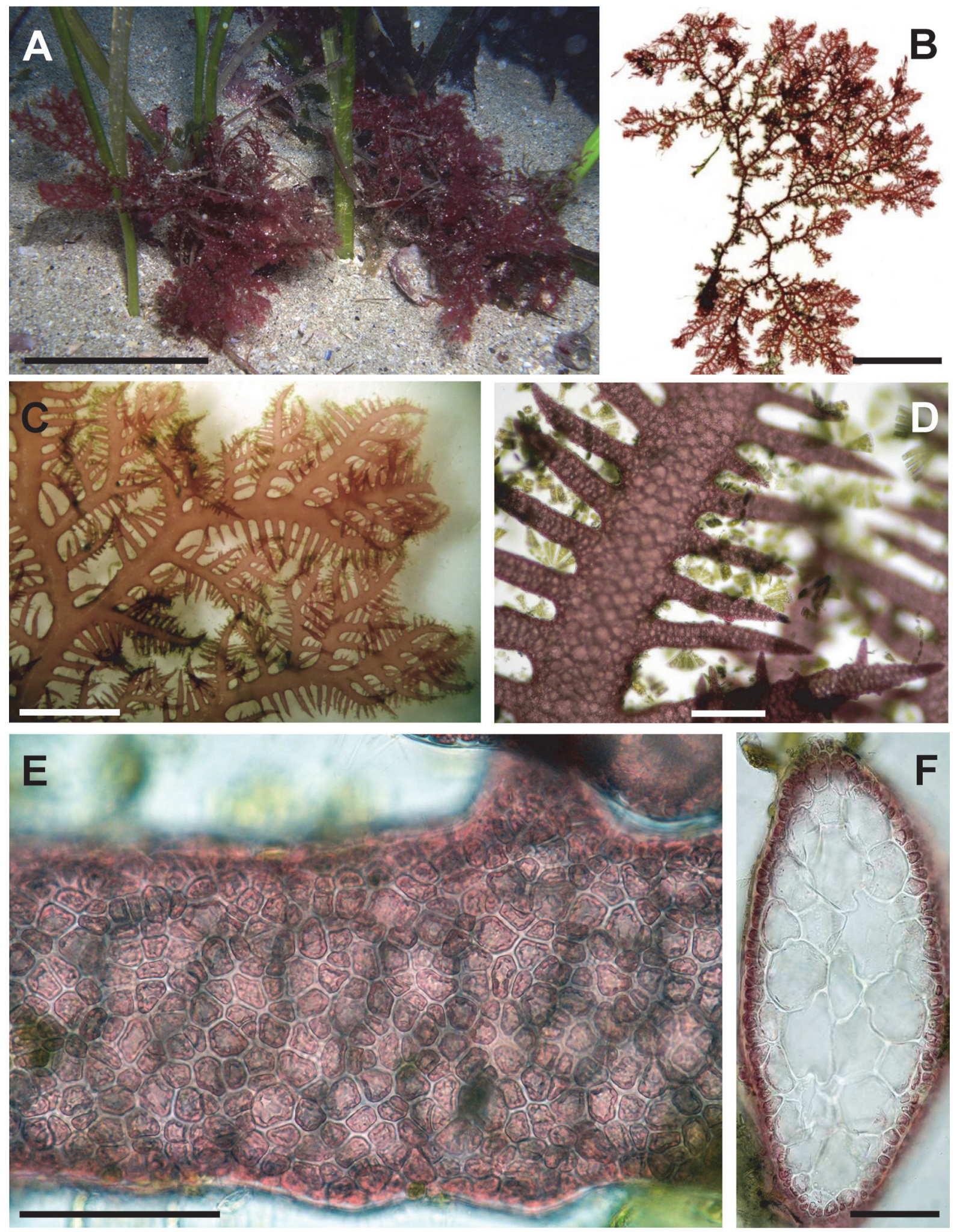

Figura 4. A-F. Plocamium cf. ovicorne, A: habitando en la base de Zostera marina, B: hábito, C, D: ramificación en series secundas alternas con numerosas proliferaciones adventicias marginales, E: células corticales en vista superficial, $\mathrm{F}$ : eje comprimido uniaxial en sección transversal. Escalas: $\mathrm{A}=5 \mathrm{~cm}, \mathrm{~B}=1 \mathrm{~cm}, \mathrm{C}=1 \mathrm{~mm}, \mathrm{D}=200 \mu \mathrm{m}, \mathrm{E}, \mathrm{F}=100 \mu$.

Figure 4. A-F. Plocamium cf. ovicorne, A: inhabiting at the base of Zostera marina, B: habit, C, D: branch in alternating secund series with numerous marginal adventitial proliferations, $\mathrm{E}$ : cortical cells in surface view, $\mathrm{F}$ : cross section of uniaxial compressed axis. Scale bars: $\mathrm{A}=5 \mathrm{~cm}, \mathrm{~B}=1 \mathrm{~cm}, \mathrm{C}=1 \mathrm{~mm}, \mathrm{D}=200 \mu \mathrm{m}, \mathrm{E}, \mathrm{F}=100 \mu$. 
\& I. Bárbara, SANT-Algae 29093, 29108, 29109, 29434, 29449, 29667, 30076, 30114, 30151, $30159,30182,30206,30250,30423,30436$, 30546. Ría de Ferrol, Torpedera, 29TNJ576125, 13-VI-2014, submareal (1-2 m) en pradera de $Z$. marina, V. García-Redondo \& I. Bárbara, SANTAlgae 29326. Ría de Ferrol, Castillo de San Felipe, 29TNJ583127, 11-V-2014, 27-I-2015, 19-III-2015, 11-IX-2015, submareal $(1 \mathrm{~m})$ en pradera de $Z$. marina, V. García-Redondo \& I. Bárbara, SANT29092, 29393, 29396, 30234. Primera cita para Europa. La morfología del material gallego es muy característica y se ajusta perfectamente a la descripción de Okamura (1896) para esta distintiva y peculiar especie. Sin embargo, el análisis molecular del material gallego mostró coincidencia con Plocamium nanum G.W. Saunders \& Lehmkuhl (Gary Saunders com. per.), por lo que son necesarios más estudios para aclarar el estatus taxonómico de $P$. cf. ovicorne.

\section{OCHROPHYTA}

Dictyota cyanoloma Tronholm, De Clerck, Gómez Garreta \& Rull Lluch

PONTEVEDRA: Ría de Vigo, Bouzas, 29TNG199747, 20-VI-2016, submareal (1 m) en pradera de Zostera marina, V. García-Redondo \& I. Bárbara, SANT-Algae 31894. Segunda cita provincial después de Bárbara et al. (2016).

\section{Dictyota spiralis Montagne (Fig. 5)}

PONTEVEDRA: Ría de Vigo, Islas Cíes, Al Este de Isla de San Martín, 29TNG081724, 19VII-2017, submareal (3-4 m) sobre roca junto a Dictyota dichotoma y $D$. dichotoma var. intricata, I. Bárbara, C. Piñeiro-Corbeira, V. Peña \& P. DíazTapia, SANT-Algae 32198. Primera cita provincial.

Dictyopteris lucida Ribera Siguán, Gómez Garreta, Pérez Ruzafa \& Barceló Martí et Rull Lluch

LUGO: Ría do Barqueiro, Punta da Cova, O Vicedo, 29TPJ679445, 30-V-2017, submareal (4 m) sobre roca en comunidad de $C$. baccata, $C$. usneoides y L. ochroleuca, I. Bárbara \& A. GarcíaFernández. SANT-Algae 31373. Segunda cita provincial después de Bárbara et al. (2014).

Pilocladus codicola (Stechell \& Gardner) Ardré

A CORUÑA: Ría de Cederia, al sur de As Blancas, 29TNJ747351, 20-IV-2016, submareal $(6 \mathrm{~m})$ sobre Codium vermilara viviendo sobre roca, I. Bárbara \& A. García-Fernández, SANTAlgae 30521. Segunda cita provincial después de Bárbara et al. (2005).

Zanardinia typus (Nardo) P.C. Silva

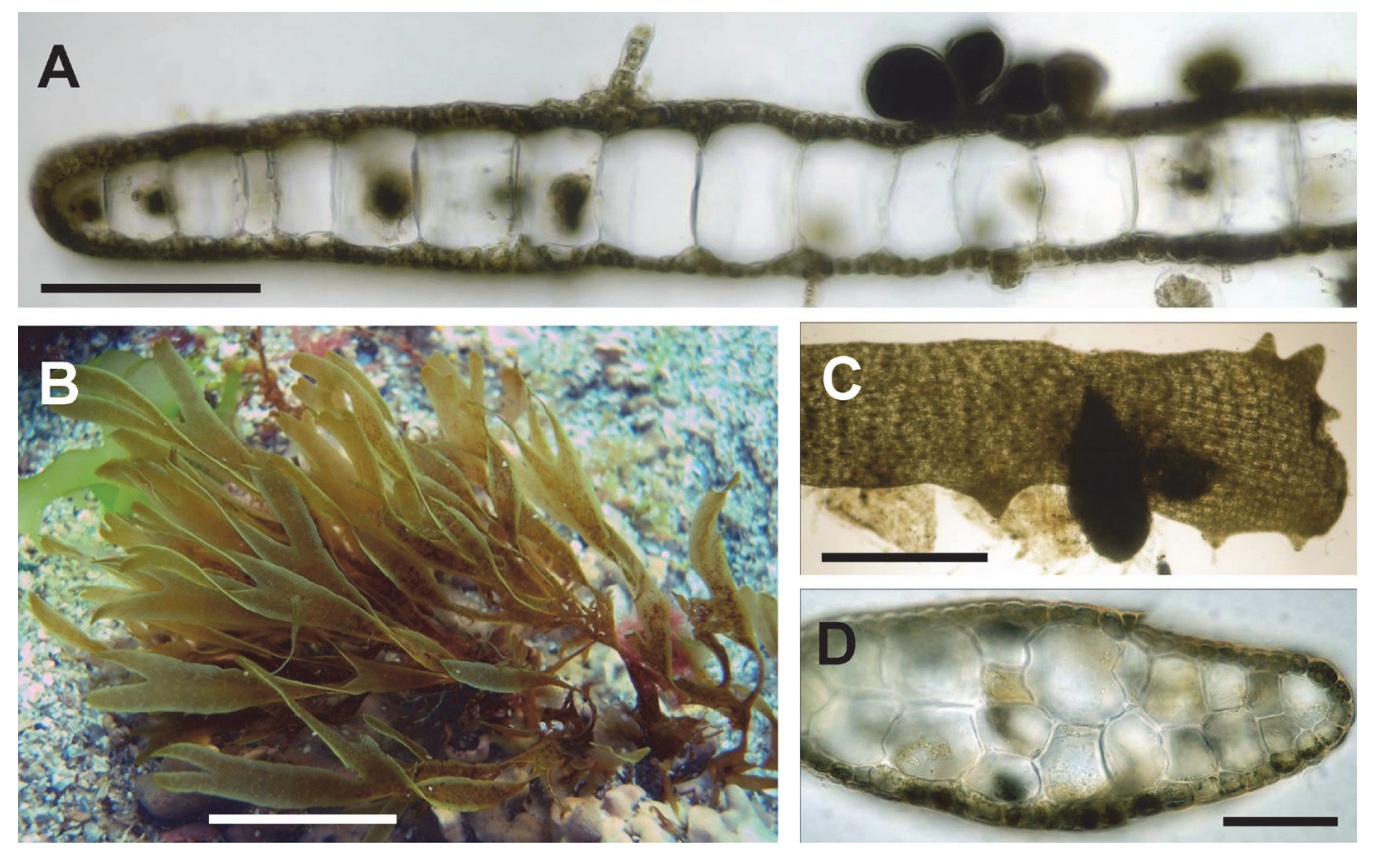

Figura 5. A-D. Dictyota spiralis, A: esporófito en sección transversal, B: hábito en el submareal, C-D: proliferación basal en vista superficial (C) y con 2-3 células medulares, sección transversal (D). Escalas: $A=200 \mu \mathrm{m}, B=5 \mathrm{~cm}, C=1 \mathrm{~mm}$, $\mathrm{D}=100 \mu \mathrm{m}$.

Figure 5. A-D. Dictyota spiralis, A: sporophyte in cross section, B: habit in the subtidal, C-D: basal blade in surface view (C), bearing 2-3 medullary cells in cross section (D). Scale bars: $A=200 \mu \mathrm{m}, B=5 \mathrm{~cm}, C=1 \mathrm{~mm}, D=100 \mu \mathrm{m}$. 

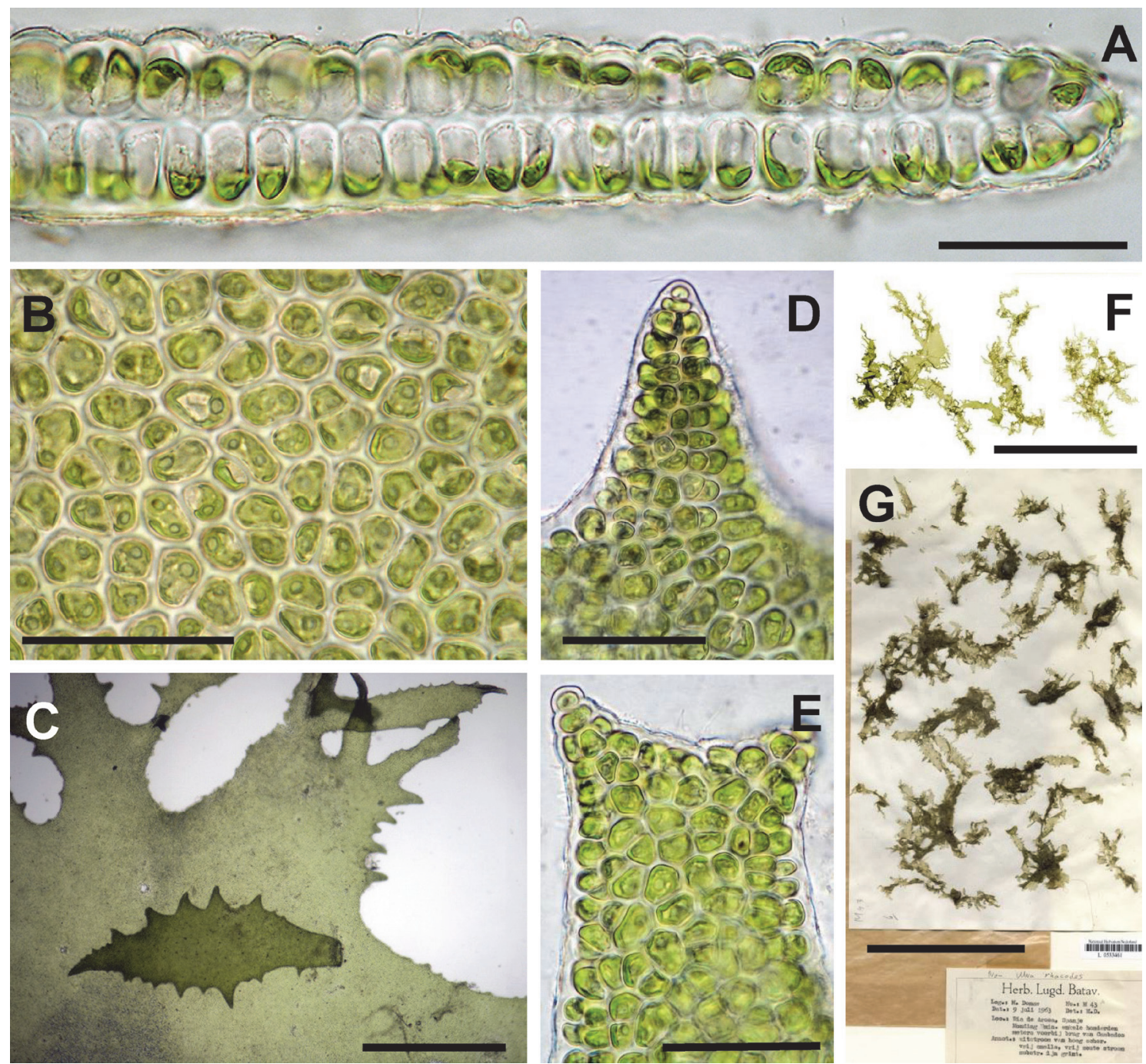

Figura 6. A-G. Ulva rhacodes, A: sección transversal de la lámina, B: vista superficial de las células con dos pirenoides, C: margen aserrado con proliferaciones, D, E: denticulaciones, F. hábito (Ría de Pontevedra, SANT-Algae 31366), G: hábito (Ría de Arosa, 9-VII-1963, M. Donze, L0533461). Escalas: A, B, D, E =50 $\mu \mathrm{m}, \mathrm{C}=1 \mathrm{~mm}, \mathrm{~F}, \mathrm{G}=10 \mathrm{~cm}$.

Figure 6. A-G. Ulva rhacodes, A: blade in cross section, B: cells with two pyrenoids in surface view, C: margin serrulate with growing tips, D, E: theeth, F: habit (Ría de Pontevedra, SANT-Algae 31366), G: habit (Ría de Arosa, 9-VII-1963, M. Donze, L-0533461). Scale bars: A, B, D, E = $50 \mu \mathrm{m}, \mathrm{C}=1 \mathrm{~mm}, \mathrm{~F}, \mathrm{G}=10 \mathrm{~cm}$.

LUGO: Ría do Barqueiro, Punta da Cova, 29TPJ679445, 30-V-2017, submareal (4 m) sobre roca en comunidad de Cystoseira baccata, $C$. usneoides y Laminaria ochroleuca, I. Bárbara \&A. García-Fernández, SANT-Algae 31374. Segunda cita provincial después de Bárbara et al. (2005b). PONTEVEDRA: Ría de Vigo, Museo del Mar, 29TNG191748, 20-VI-2016, submareal (1 m) bajo pradera de Z. marina, V. García-Redondo \& I. Bárbara, SANT-Algae 31821. Segunda cita provincial después de Niell (1976).

\section{CHLOROPHYTA}

Derbesia marina (Lyngbye) Solier como Halicystis ovalis (Lyngbye) J.E. Areschoug stadium PONTEVEDRA: Ría de Vigo, Islas Cíes, Islas Cíes, Islote Viños, 29TNG084737, 3-V-2018, submareal (3 $\mathrm{m}$ ) sobre roca, bajo Padina pavonica, I. Bárbara, C. Piñeiro Corbeira, V. Peña \& P. DíazTapia, SANT-Algae 32312. Segunda cita provincial después de Bárbara et al. (2016).

Ulva rhacodes (Holmes) Papenfuss (Fig. 6)

PONTEVEDRA: Ría de Pontevedra, Placeres, 29TNG263959, 19-VII-2016, submareal (1 m), suelta junto a Zostera marina, Z. noltei y U. rigida, V. García-Redondo \& I. Bárbara, SANT-Algae 31366, 31367. Segunda cita provincial y para Galicia después de Hoek \& Donze (1966) y Donze (1968). 

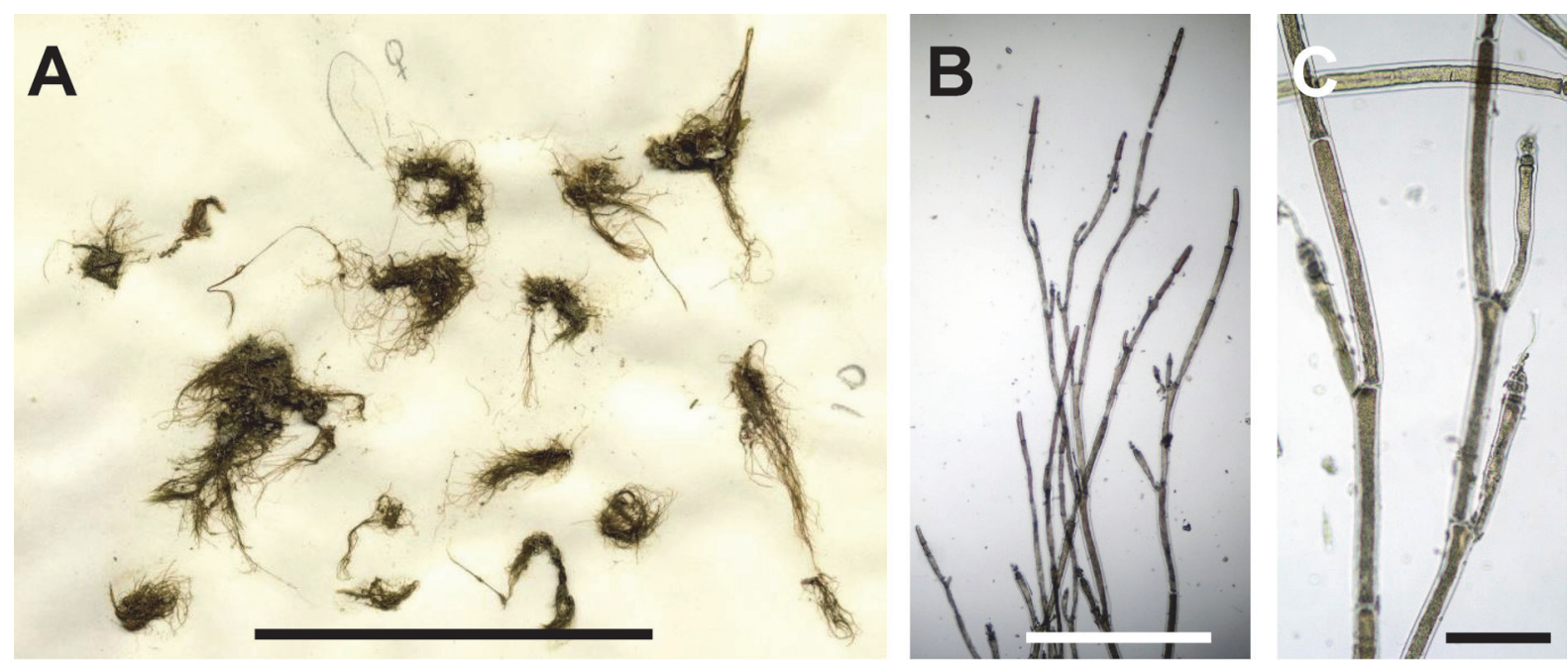

Figura 7. A-C. Tiffaniella capitata, A: hábito (como Spermothamnion strictum, Ría de Arousa, 29-I-1964, M. Donze, submareal, L-0533447), B, D: talos femeninos con carpogonios. Escalas: $\mathrm{A}=5 \mathrm{~cm}, \mathrm{~B}=1 \mathrm{~mm}, \mathrm{C}=200 \mu \mathrm{m}$.

Figure 7. A-C. Tiffaniella capitata, A: habit (as Spermothamnion strictum, Ría de Arousa, 29-I-1964, M. Donze, subtidal, L-0533447), B, C: female plants with carpogonia. Scale bars: $A=5 \mathrm{~cm}, B=1 \mathrm{~mm}, C=200 \mu \mathrm{m}$.
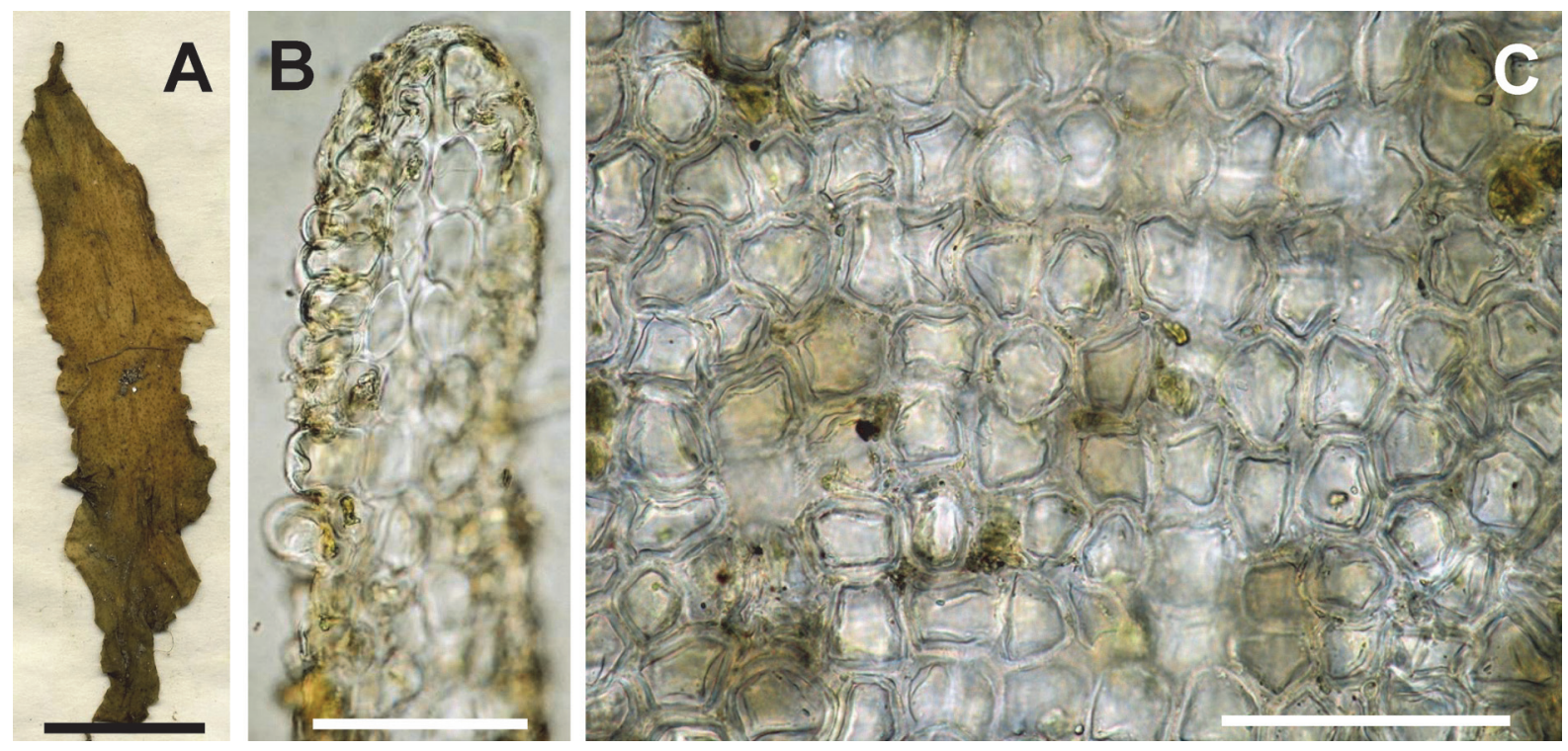

Figura 8. A-C. Punctaria latifolia, A: hábito (como P. plantaginea, Ría de Arosa, 2-VII-1963, M. Donze, subtidal, L-0533460), B: sección transversal; C: vista superficial. Escalas: $\mathrm{A}=2 \mathrm{~cm}, \mathrm{~B}, \mathrm{C}=100 \mu \mathrm{m}$.

Figure 8. A-C. Punctaria latifolia, A: habit (as P. plantaginea, Ría de Arosa, 2-VII-1963, M. Donze, subtidal, L-0533460), B: cross section; C: surface view. Scale bars: $A=2 \mathrm{~cm}, \mathrm{~B}, \mathrm{C}=100 \mu \mathrm{m}$.

\section{CORRECCIONES Y TAXA EXCLUDENDA}

\section{Spermothamnion strictum (C. Agardh) Ardissone}

(Fig. 7)

El único registro conocido de esta especie para Galicia es el de Donze (1968), recolectado en la ría de Arosa (Preguntorio, 28-I-1964, laguna litoral, L-0533448; 29-I-1964, submareal, L-0533447). Los pliegos estudiados corresponden con Tiffaniella capitata (Schousboe ex Bornet) Doty et Meñez, especie descrita en Bárbara et al. (1992) y DíazTapia \& Bárbara (2013). Por lo tanto, la cita de $S$. 
strictum debe ser excluida de la flora de Galicia.

Punctaria plantaginea (Roth) Greville (Fig. 8)

El único registro conocido de esta especie para Galicia es el de Donze (1968),recolectado en la ría de Arosa (2-VII-1963, L-0533460). El pliego estudiado corresponde con Punctaria latifolia Greville, especie registrada en varias localidades gallegas (Bárbara et al. 2005a). Por lo tanto, la cita de $P$. plantaginea debe ser excluida de la flora de Galicia.

\section{Agradecimientos}

Las muestras del archipiélago de Cíes han sido recolectadas en el marco del proyecto HIPPOPARQUES (1541S/2015), financiado por el Organismo Autónomo Parques Nacionales (Gobierno de España, Ministerio de Agricultura, Alimentación y Medio Ambiente). Los autores agradecen a Xunta de Galicia la concesión de la ayuda (GPC2015/025), V. Peña la financiación del programa postdoctoral Campus Industrial de Ferrol (Universidade da Coruña) y P. Díaz-Tapia al programa postdoctoral de la Xunta de Galicia.. Finalmente, agradecemos las sugerencias y correcciones de los revisores y el equipo editorial.

\section{Referencias}

Bañón, R. (Ed.) (2017). Inventario de la biodiversidad marina de Galicia: Proyecto LEMGAL. Consellería do Mar, Xunta de Galicia, Santiago de Compostela. 570 pp.

Bárbara, I., Cremades, J. Calvo, S. López Rodríguez M.C. \& Dosil J. (2005a). Checklist of the benthic marine and brackish Galician algae (NW Spain). Anales Jard. Bot. Madrid, 62(1), 69-100.

Bárbara, I., Cremades, J. \& López Rodríguez, M.C. (1992). Tiffaniella capitatum (Ceramiaceae, Rhodophyta) en las costas atlánticas de Europa. Cryptogamie-Algologie, 13(4), 291-299.

Bárbara, I., De Clerck, O. García-Redondo, V., Peña, V. García-Fernández, A., Peteiro, C. \& Sánchez, N. (2015). Nuevas citas y adiciones corológicas para la flora bentónica marina del Atlántico Ibérico. Acta Botánica Malacitana, 40, 191-198.

Bárbara, I. Díaz, P., Araújo, R., Peña, V., Berecibar, E., Cremades, J., Freire, Ó., Baamonde, S., Novo, T., Calvo, S., López Rodríguez, M.C., Afonso-Carrillo, J., De Clerck, O., Santos, R., Sousa-Pinto, I., Tibaldo, M., Lagos, V., López, C., Secilla, A., Santolaria, A., Díez, I., \& Veiga, A.J. (2006). Adiciones corológicas y correcciones a la flora bentónica marina del norte de la Península Ibérica. Nova Acta Científica Compostelana, 15, 77-88.

Bárbara, I., Díaz, P., Cremades, J., Tibaldo, M., Freire, Ó., Peña, V., Lagos, V. Calvo, S., Veiga, A.J., Peteiro, C., López Rodríguez, M.C. \&Araújo, R. (2005b). Adiciones corológicas a la flora bentónica marina del norte de la Península Ibérica. Nova Acta Científica Compostelana (Bioloxía), 14, 83-88.
Bárbara, I., Díaz, P., Peña, V., Freire, Ó., Baamonde, S., Cremades, J., Lagos, V. \& Lema, C.(2008). Adiciones corológicas a la flora bentónica marina de Galicia. Nova Acta Cientifica Compostelana (Bioloxía), 17, 169-175.

Bárbara, I., Díaz Tapia, P., Peteiro, C., Berecibar, E., Peña, V., Sánchez, N., Tavares, A.M., Santos, R., Secilla, A., Riera Fernández, P., Bermejo R., \& García, V. (2012). Nuevas citas y aportaciones corológicas para la flora bentónica marina del Atlántico de la Península Ibérica. Acta Botánica Malacitana, 37, 5-32.

Bárbara, I., Peña, V., García-Redondo, V., Díaz Tapia, P., García-Fernández, A., Lugilde, J. \& CorbeiraFernández, C. (2016). Nuevas citas y registros corológicos para la flora bentónica marina del Noroeste Ibérico. Acta Botánica Malacitana, 41, 247-289.

Bárbara, I., Peteiro, C., Peña, V., Altamirano, M., Piñeiro-Corbeira, C., Sánchez, N., Díaz-Tapia, P., García-Redondo, V., García-Fernández, A \& Zanolla-Balbuena, M. (2014). Adiciones florísticas y aportaciones corológicas para la flora bentónica marina del atlántico ibérico. Acta Botánica Malacitana, 39, 207-216.

Calvo, S., Bárbara, I. \& Cremades, J. (1999). Benthic algae of saltmarshes (Corrubedo Natural Park, NW Spain): The Flora. Botanica Marina, 42, 343-353.

Cremades, J., Bárbara, I., Granja, A. \& Veiga, A.J. (1997). Fragmenta Chorologica Occidentalia, Algae, 5957-6058. Anales Jard. Bot. Madrid, 55(2): 433-440.

Díaz Tapia, P. (2014). Actualización del catálogo de algas marinas exóticas de Galicia. Memoria Final Bolsas de Investigación de la Diputación de A Coruña (ined.). $53 \mathrm{pp}$.

Díaz-Tapia, P. \& Bárbara, I. (2013). Seaweeds from sandcovered rocks of the Atlantic Iberian Peninsula. Part 1. The Rhodomelaceae (Ceramiales, Rhodophyta). Cryptogamie-Algologie, 34(4), 352-422.

Donze, M. (1968). The algal vegetation of the Ría de Arosa (NW. Spain). Blumea 16, 159-192.

Gallardo, T., Bárbara, I., Afonso-Carrillo, J., Bermejo,R., Altamirano, M., GómezGarreta, A., BarcelóMartí, M . C ., Rull Lluch, J., Ballesteros, E. \& De La Rosa, J. (2016). Nueva lista crítica de las algas bentónicas marinas de España. ALGAS, Boletín Informativo Sociedad Española Ficología, 51, 7-52.

Hoek, C. van den \& Donze, M. (1966). A contribution to the knowledge of Ulva rhacodes (Chlorophyceae Ulotrichales). Nova Hedwigia, 10(3/4), 495-498, Tabs. 144-149.

López Rodríguez, M.C. \& Pérez-Cirera, J.L. (1996). Cianófitos en medios alterados por la contaminación industrial en el noroeste de la Península Ibérica. Anales del Jardín Botánico de Madrid, 54, 43-49.

Niell, F.X. (1976). La distribución de Zanardinia prototypus (Nardo) en la Península Ibérica. Abstract Simposio Conmemorativo del Centenario de Lagasca, 22.

Okamura, K. (1896). Contribution to knowledge of the marine algae of Japan, II. Botanical Magazine, Tokyo, 10, 21-28, 33-40.

Won, B.Y., Cho, T.O. \& Fredericq, S. (2009). Morphological and molecular characterization of species of the genus Centroceras (Ceramiaceae, Ceramiales), including two new species. Journal of Phycology, 45:227-50. 